\title{
Boundedness of Marcinkiewicz Integrals on RBMO Spaces over Nonhomogeneous Metric Measure Spaces
}

\author{
Ji Cheng and Guanghui Lu \\ College of Mathematics and System Sciences, Xinjiang University, Urumqi 830046, China \\ Correspondence should be addressed to Guanghui Lu; lghwmm1989@126.com
}

Received 23 October 2014; Revised 13 February 2015; Accepted 23 February 2015

Academic Editor: Mark J. Friedman

Copyright (C) 2015 J. Cheng and G. Lu. This is an open access article distributed under the Creative Commons Attribution License, which permits unrestricted use, distribution, and reproduction in any medium, provided the original work is properly cited.

Let $(\mathscr{X}, d, \mu)$ be a metric measures space satisfying the upper doubling conditions and the geometrically doubling conditions in the sense of Hytönen. Under the assumption that the dominating function satisfies the weak reverse doubling condition, the authors prove that Marcinkiewicz integral with kernel satisfying certain stronger Hörmander-type condition is bounded on $\mathrm{RBMO}(\mu)$ space.

\section{Introduction}

We all know that the Littlewood-Paley $g$-function has been playing an important role in harmonic analysis and the Marcinkiewicz integral is an essential Littlewood-Paley $g$-function. As an analogy to Littlewood-Paley $g$-function without going into the interior of the unit disk, in 1938, Marcinkiewicz introduced the integral on one dimensional Euclidean space $\mathbb{R}$, which now is called the Marcinkiewicz integral, and conjectured that it is bounded on $L^{p}([0,2 \pi])$ for $p \in$ $(1, \infty)$ (see [1]). In 1944, Zygmund proved the Marcinkiewicz conjecture by using the complex variable method in [2]. Particularly, in 1958, Stein introduced the higher dimensional Marcinkiewicz integral (see [3]). Let $\Omega$ be homogeneous of degree zero in $\mathbb{R}^{n}$ for $n \geq 2$ and integrable and have mean value zero on the unit sphere $\mathbb{S}^{n-1}$. The higher dimensional Marcinkiewicz integral $\mathscr{M}_{\Omega}$ is defined by

$$
\begin{aligned}
& \mathscr{M}_{\Omega}(f)(x) \\
& :=\left(\int_{0}^{\infty}\left|\int_{|x-y| \leq t} \frac{\Omega(x-y)}{|x-y|^{n-1}} f(y) \mathrm{d} y\right|^{2} \frac{\mathrm{d} t}{t^{3}}\right)^{1 / 2}, \quad x \in \mathbb{R}^{n} .
\end{aligned}
$$

Recently, many papers focus on the boundedness of this operator on various function spaces. We refer the reader to see $[4,5]$.
Many results from real analysis and harmonic analysis on the classical Euclidean spaces have been extended to the space of homogeneous type by Coifman and Weiss in [6]. Recall that a metric space $(\mathscr{X}, d)$ equipped with a Borel measure $\mu$ is called a space of homogeneous type, if $(\mathscr{X}, d, \mu)$ satisfies the following doubling measure condition that there exists a positive constant $C_{\mu}$ such that, for all balls $B(x, r):=\{y \in \mathscr{X}$ : $d(x, y)<r\}$ with $x \in \mathscr{X}$ and $r \in(0, \infty)$,

$$
\mu(B(x, 2 r)) \leq C_{\mu} \mu(B(x, r)) .
$$

Meanwhile, many classical results concerning the theory of Calderón-Zygmund operators and function spaces have been proved still valid for nondoubling measures. In particular, let $\mu$ be a nonnegative Radon measure on $\mathbb{R}^{n}$ which only satisfies the polynomial growth condition that there exists a positive constant $C_{0}$ and $d \in(0, n]$ such that, for all $x \in \mathbb{R}^{n}$ and $r \in(0, \infty)$,

$$
\mu(B(x, 2 r)) \leq C_{0} r^{d},
$$

where $B(x, r):=\left\{y \in \mathbb{R}^{n}:|x-y|<r\right\}$. Such a measure $\mu$ need not satisfy the doubling condition (2). The analysis with nondoubling measures plays an important role in solving the longstanding open Painlevé problem by Tolsa in [7]. In 2007, $\mathrm{Hu}$ et al. introduced the Marcinkiewicz integral with nondoubling measures and got some excellent results in [8]. In 2012, Wang et al. established the boundedness of Marcinkiewicz integrals on $\mathrm{RBMO}(\mu)$ in [9]. 
However, in 2010, Hytönen pointed out that the measures satisfying the polynomial growth condition are different from the doubling measures in [10]. Hytönen introduced a new class of metric measure spaces which satisfy the upper doubling condition and the geometrically doubling condition (resp., see Definitions 1 and 3 below). This new class of metric measure space is called the nonhomogeneous metric measure space, which includes both spaces of homogeneous type and metric spaces with the measures satisfying (3) as special cases. We refer the reader to the monograph [11] for several recent developments on harmonic analysis in this setting.

In this paper, we mainly discuss the boundedness of Marcinkiewicz integrals $\mathscr{M}$ on $\operatorname{RBMO}(\mu)$ with nonhomogeneous metric measure spaces. Now we recall some notations and definitions as follows.

Definition 1. A metric measure space $(\mathscr{X}, d, \mu)$ is said to be upper doubling, if $\mu$ is Borel measure on $\mathscr{X}$ and there exists a dominating function $\lambda: \mathscr{X} \times(0, \infty) \rightarrow(0, \infty)$ and a positive constant $C_{\lambda}$ such that, for each $x \in \mathscr{X}: r \rightarrow \lambda(x, r)$, is nondecreasing and, for all $x \in \mathscr{X}$ and $r \in(0, \infty)$,

$$
\mu(B(x, r)) \leq \lambda(x, r) \leq C_{\lambda} \lambda\left(x, \frac{r}{2}\right) .
$$

Remark 2. (1) Obviously, a space of homogeneous type is a special case of upper doubling spaces, where one can take the dominating function $\lambda(x, r)=\mu(B(x, r))$. Moreover, let $\mu$ be a nonnegative Radon measure on $\mathbb{R}^{n}$ which only satisfies the Polynomial growth condition. By taking $\lambda(x, r)=C r^{n}$, we see that $\left(\mathbb{R}^{n},|\cdot|, \mu\right)$ is also an upper doubling measure space.

(2) It was proved that there exists a dominating function $\tilde{\lambda}$ related to $\lambda$ satisfying the property that there exists a positive constant $C_{\tilde{\lambda}}$ such that $\tilde{\lambda} \leq \lambda, C_{\tilde{\lambda}} \leq C_{\lambda}$ and, for all $x, y \in \chi$ with $d(x, y) \leq \lambda$,

$$
\tilde{\lambda}(x, r) \leq C_{\widetilde{\lambda}} \tilde{\lambda}(y, r) .
$$

Based on this, in this paper, we always assume that the dominating function $\lambda$ also satisfies (5).

Definition 3. A metric space $(\mathscr{X}, d)$ is said to be geometrically doubling, if there exist some $N_{0} \in \mathbb{N}=\{1,2, \ldots\}$ such that, for any ball $B(x, r) \subset \mathscr{X}$, there exists a finite ball covering $\left\{B\left(x_{i}, r / 2\right)\right\}_{i}$ of $B(x, r)$ such that the cardinality of this covering is at most $N_{0}$.

Remark 4. Let $(\mathscr{X}, d)$ be a metric space. Hytönen showed that the following statements are mutually equivalent (see [10]):

(1) $(\mathscr{X}, d)$ is geometrically doubling.

(2) For any $\epsilon \epsilon(0,1)$ and ball $B(x, r) \subset \mathscr{X}$, there exists a finite ball covering $\left\{B\left(x_{i}, \epsilon r\right)\right\}_{i}$ of $B(x, r)$ such that the cardinality of this covering is at most $N \epsilon^{-n}$. Here and in what follows, $N_{0}$ is as in Definition 3 and $n=$ $\log _{2} N_{0}$.

(3) For every $\epsilon \in(0,1)$, any ball $B(x, r) \subset \mathscr{X}$ can contain at most $N \epsilon^{-n}$ centers $\left\{x_{i}\right\}_{i}$ of disjoint balls with radius $\epsilon r$.
(4) There exists $M \in \mathbb{N}$ such that any ball $B(x, r) \subset \mathscr{X}$ can contain at most $N \epsilon^{-n}$ centers $\left\{x_{i}\right\}_{i}$ of disjoint balls $\left\{B\left(x_{i}, r / 4\right)\right\}_{i=1}^{M}$.

Definition 5. For any two balls $B \subset S$, define

$$
K_{B, S}=1+\int_{2 S \backslash B} \frac{1}{\lambda\left(c_{B}, d\left(x, c_{B}\right)\right)} \mathrm{d} \mu(x),
$$

where above and in that follows, for a ball $B=B\left(c_{B}, r_{B}\right)$ and $\rho>0, \rho B=B\left(c_{B}, \rho r_{B}\right) . c_{B}$ is the center of ball $B$.

Remark 6. The following discrete version, $\widetilde{K}_{B, S}$, of $K_{B, S}$ defined in Definition 5 was first introduced by Bui and Duong in nonhomogeneous metric measure spaces (see [12]), which is more close to the quantity $K_{\mathrm{Q}, R}$ introduced by Tolsa [13] in the setting of nondoubling measures. For any two balls $B \subset S$, let $\widetilde{K}_{B, S}$ be defined by

$$
\widetilde{K}_{B, S}:=1+\sum_{k=1}^{N_{B, S}} \frac{\mu\left(6^{k} B\right)}{\lambda\left(c_{B}, 6^{k} r_{B}\right)},
$$

where $r_{B}$ and $r_{S}$, respectively, denote the radius of the balls $B$ and $S$ and $N_{B, S}$ is the smallest integer satisfying $6^{N_{B, S}} r_{B} \geq r_{S}$. Obviously, $K_{B, S} \leq C \widetilde{K}_{B, S}$. That was pointed by Bui and Duong in [12]; in general, it is not true that $K_{B, S} \sim C \widetilde{K}_{B, S}$.

Definition 7. Let $\alpha, \beta \in(0, \infty)$. A ball $B \subset \mathscr{X}$ is called $(\alpha, \beta)$ doubling if $\mu(\alpha B) \leq \beta \mu(B)$.

It was proved in [10] that if a metric measure space $(\mathscr{X}, d, \mu)$ is upper doubling and $\beta>C_{\lambda}^{\log _{2} \alpha}=\alpha^{\nu}$, then, for every ball $B \subset \mathscr{X}$, there exists some $j \in \mathbb{Z}_{+}$such that $\alpha^{j} \beta$ is $(\alpha, \beta)$-doubling. Moreover, let $(\mathscr{X}, d)$ be geometrically doubling, $\beta>\alpha^{n}$ with $n=\log _{2} N_{0}$, and $\mu$ is Borel measure on $\mathscr{X}$ which is finite on bounded sets. In [10] Hytönen also showed that, for $\mu$-almost every $x \in \mathscr{X}$, there exist arbitrarily small $(\alpha, \beta)$-doubling balls centered at $x$. Furthermore, the radius of these balls may be chosen to be form $\alpha^{-j} r$ for $j \in \mathbb{N}$ and any preassigned number $r \in(0, \infty)$. Throughout this paper, for any $\alpha \in(1, \infty)$ and ball $B, \widetilde{B}$ denotes the smallest $\left(\alpha, \beta_{\alpha}\right)$-doubling ball of the form $\alpha^{j} B$ with $j \in \mathbb{Z}_{+}$, where

$$
\begin{aligned}
\beta_{\alpha} & =\max \left\{\alpha^{3 n}, \alpha^{3 \nu}\right\}+30^{n}+30^{\nu} \\
& =\alpha^{3(\max (n, v))}+30^{n}+30^{\nu}
\end{aligned}
$$

If $\alpha=6$, we denote the ball $\widetilde{B}^{\alpha}$ simply by $\widetilde{B}$.

Let $K(x, y)$ be a $\mu$-locally integrable function on $\mathscr{X} \times \mathscr{X} \backslash$ $\{(x, x): x \in \mathscr{X}\}$. Assume that there exists a positive constant $C$ such that, for any $x, y \in \mathscr{X}$ with $x \neq y$,

$$
|K(x, y)| \leq C \frac{d(x, y)}{\lambda(x, d(x, y))},
$$


and, for any $x, y, y^{\prime} \in \mathscr{X}$,

$$
\begin{aligned}
& \int_{d(x, y) \geq 2 d\left(y, y^{\prime}\right)}\left[\left|K(x, y)-K\left(x, y^{\prime}\right)\right|\right. \\
&\left.+\left|K(y, x)-K\left(y^{\prime}, x\right)\right|\right] \frac{1}{d(x, y)} \mathrm{d} \mu(x) \leq C .
\end{aligned}
$$

The Marcinkiewicz integral $\mathscr{M}(f)$ associated with the above kernel $K(x, y)$ is defined by

$$
\begin{aligned}
& \mathscr{M}(f)(x) \\
& \quad=\left(\int_{0}^{\infty}\left|\int_{d(x, y) \leq t} K(x, y) f(y) \mathrm{d} \mu(y)\right|^{2} \frac{\mathrm{d} t}{t^{3}}\right)^{1 / 2}, \quad x \in \mathscr{X} .
\end{aligned}
$$

Obviously, by taking $\lambda(x, r)=C r^{n}$, we see that, in the classical Euclidean space $\mathbb{R}^{n}$, if

$$
K(x, y)=\frac{\Omega(x-y)}{|x-y|^{n-1}},
$$

with $\Omega$ homogeneous of degree zero and $\Omega \in \operatorname{Lip}_{\alpha}\left(\mathbb{S}^{d-1}\right)$ for some $\alpha \in(0,1]$, then $K(x, y)$ satisfies (5) and (8). In 2014, Lin and Yang [14] established the equivalent boundedness of Marcinkiewicz integral $\mathscr{M}$ with kernel $K(x, y)$ satisfying (9) and (10). In this note, we make some modification for the kernel. Besides satisfying the regular condition (9), $K(x, y)$ also satisfies that, for any $x, x^{\prime}$ and $y \in \mathscr{X}$ with $d(x, y) \geq$ $2 d\left(x, x^{\prime}\right)$, there exists $0<\delta \leq 1$ such that

$$
\begin{aligned}
& \left|K(x, y)-K\left(x^{\prime}, y\right)\right|+\left|K(y, x)-K\left(y, x^{\prime}\right)\right| \\
& \quad \leq \frac{\left[d\left(x, x^{\prime}\right)\right]^{\delta}[d(x, y)]^{1-\delta}}{\lambda(x, d(x, y))},
\end{aligned}
$$

and, for any $x \in \mathscr{X}$ and any two positive real numbers $\varepsilon, N$ with $\varepsilon<N$,

$$
\int_{\varepsilon<d(x, y)<N} K(x, y) \mathrm{d} \mu(y)=0 .
$$
(10).

When the kernel satisfies condition (13), it also satisfies

Definition 8. Let $\eta \in(1, \infty)$. A function $f \in L_{\text {loc }}^{1}(\mu)$ is said to be the space $\operatorname{RBMO}(\mu)$, if there exists a positive constant $C$ and a number $f_{B}$ for any ball $B$ such that, for all balls $B$,

$$
\frac{1}{\mu(\eta B)} \int_{B}\left|f(y)-f_{B}\right| \mathrm{d} \mu(y) \leq C
$$

and, for balls $B \subset S$,

$$
\left|f_{B}-f_{S}\right| \leq C K_{B, S}
$$

Moreover, the norm of $f$ in $\operatorname{RBMO}(\mu)$ is defined to be the minimal constant $C$ as above and denoted by $\|f\|_{\operatorname{RBMO}(\mu)}$.
Definition 9. A dominating function $\lambda$ is said to satisfy the weak reverse doubling condition if, for all $r \in(0,2 \operatorname{diam}(\mathscr{X}))$ and $a \in(1,2 \operatorname{diam}(\mathscr{X}) / r)$, there exists a number $C(a) \epsilon$ $[1, \infty)$, depending only on $a$ and $\mathscr{X}$, such that, for all $x \in \mathscr{X}$,

$$
\lambda(x, a r) \geq C(a) \lambda(x, r)
$$

and, moreover,

$$
\sum_{k=1}^{\infty} \frac{1}{C\left(a^{k}\right)}<\infty
$$

Remark 10. If the dominating function $\lambda$ satisfies the weak reverse doubling condition, then $K_{B, S} \sim \widetilde{K}_{B, S}$ for any two balls; see [15].

Throughout this paper, we denote by $C$ a positive constant which is independent of the main parameters involved, but it may be different from line to line. For any $p \in[1, \infty]$, we denote by $p^{\prime}$ its conjugate index; namely, $1 / p+1 / p^{\prime}=1$.

\section{Main Result and Its Proof}

We give the main result as follows.

Theorem 11. Let $(\mathscr{X}, d, \mu)$ be a nonhomogeneous metric measure space with the dominating function satisfying the weak reverse doubling condition, let $K(x, y)$ satisfy conditions (9), (13), and (14), and let $\mathscr{M}$ be defined as (11). If $\mathscr{M}$ is bounded on $L^{2}(\mu)$, then, for any $f \in R B M O(\mu)$,

$$
\|\mathscr{M}(f)\|_{R B M O(\mu)} \leq C\|f\|_{R B M O(\mu)},
$$

where $C$ is a positive constant independent of $f$, only when $\mathscr{M}(f)$ is finite $\mu$-almost everywhere; otherwise, $\mathscr{M}(f) \notin$ $R B M O(\mu)$.

In order to prove the theorem, we need the following two lemmas. The following useful properties of $K_{B, S}$ were proved in $[10,16]$; see also [17].

Lemma 12. (i) For all balls $B \subset R \subset S, K_{B, R} \leq K_{R, S}$.

(ii) For any $\rho \in[1, \infty)$, there exists a positive constant $C$, depending on $\rho$, such that, for all balls $B \subset S$ with $r_{S} \leq \rho r_{B}$, $K_{B, S} \leq$ C.

(iii) For any $\alpha \in[1, \infty)$, there exists a positive constant $\widetilde{C}$, depending on $\alpha$, such that, for all balls $B, K_{B, \widetilde{B}^{\alpha}} \leq \widetilde{C}$.

(iv) There exists a positive constant $c$ such that, for all balls $B \subset R \subset S, K_{B, S} \leq K_{B, R}+c K_{R, S}$. In particular, if $B$ and $R$ are concentric, then $c=1$.

(v) There exists a positive constant $\widetilde{c}$ such that, for all balls $B \subset R \subset S, K_{R, S} \leq \widetilde{c} K_{B, S}$; moreover, if $B$ and $R$ are concentric, then $K_{R, S} \leq K_{B, S}$.

We also need the following characterization of $\operatorname{RBMO}(\mu)$; see [16, Proposition 2.10] and [18, Lemma 3.2].

Lemma 13. Let $\alpha \in(1, \infty)$, and let $\beta_{\alpha}$ be as in (8). For $f \in$ $L_{l o c}^{1}(\mu)$, the following statements are equivalent: 
(i) $f \in R B M O(\mu)$;

(ii) there exists a nonnegative constant $C$ such that, for all $\left(\alpha, \beta_{\alpha}\right)$-doubling balls $B$,

$$
\frac{1}{\mu(B)} \int_{B}\left|f(y)-m_{B}(f)\right| d \mu(y) \leq C,
$$

and that, for all $\left(\alpha, \beta_{\alpha}\right)$-doubling balls $B \subset S$,

$$
\left|m_{B}(f)-m_{S}(f)\right| \leq C K_{B, S},
$$

where and in what follows $m_{B}(f)=(1 / \mu(B)) \int_{B} f(y) d \mu(y)$.

Moreover, the minimal constant $C$ as above is equivalent to $\|f\|_{\operatorname{RBMO}(\mu)}$.

Proof of Theorem 11. For $f \in \operatorname{RBMO}(\mu)$, we have that $\mathscr{M}(f)$ is finite $\mu$-almost everywhere and decompose

$$
\begin{aligned}
f(x) & =\left(f(x)-f_{6 S}\right) \chi_{6 S}(x)+\left(f(x)-f_{6 S}\right) \chi_{X \backslash 6 S}(x)+f_{6 S} \\
& :=f_{1}(x)+f_{2}(x)+f_{3}
\end{aligned}
$$

for any two balls $B \subset S$.

By the vanishing (14), we have

$$
\begin{aligned}
& \int_{d(x, y) \leq t} K(x, y) f_{3} \mathrm{~d} \mu(y) \\
& =\lim _{\varepsilon \rightarrow 0} \int_{\varepsilon<d(x, y) \leq t} K(x, y) f_{3} \mathrm{~d} \mu(y)=0 .
\end{aligned}
$$

Furthermore,

$\mathscr{M}\left(f_{3}\right)(x)=\left(\int_{0}^{\infty}\left|\int_{d(x, y) \leq t} K(x, y) f_{3} \mathrm{~d} \mu(y)\right|^{2} \frac{\mathrm{d} t}{t^{3}}\right)^{1 / 2}=0$

By applying the Minkowski inequality and vanishing condition (14), we have

$$
\begin{aligned}
& \mathscr{M}(f)(x) \leq \mathscr{M}\left(f_{1}\right)(x)+\mathscr{M}\left(f_{2}\right)(x), \\
& \mathscr{M}\left(f_{2}\right)(x) \leq \mathscr{M}\left(f_{1}\right)(x)+\mathscr{M}(f)(x) .
\end{aligned}
$$

Then it is easy to get that, for any $x, y \in \mathscr{X}$,

$$
\begin{aligned}
& \mathscr{M}(f)(x)-\mathscr{M}(f)(y) \leq \mathscr{M}\left(f_{1}\right)(x) \\
& \quad+\mathscr{M}\left(f_{2}\right)(x)-\mathscr{M}\left(f_{2}\right)(y)+\mathscr{M}\left(f_{1}\right)(y), \\
& \mathscr{M}(f)(y)-\mathscr{M}(f)(x) \leq \mathscr{M}\left(f_{1}\right)(y) \\
& \quad+\mathscr{M}\left(f_{2}\right)(y)-\mathscr{M}\left(f_{2}\right)(x)+\mathscr{M}\left(f_{1}\right)(x),
\end{aligned}
$$

which follows that

$$
\begin{aligned}
&|\mathscr{M}(f)(x)-\mathscr{M}(f)(y)| \\
& \leq \mathscr{M}\left(f_{1}\right)(x) \\
& \quad+\left|\mathscr{M}\left(f_{2}\right)(x)-\mathscr{M}\left(f_{2}\right)(y)\right|+\mathscr{M}\left(f_{1}\right)(y) .
\end{aligned}
$$

So, we deduce

$$
\begin{aligned}
& \int_{B} \int_{S}|\mathscr{M}(f)(x)-\mathscr{M}(f)(y)| \mathrm{d} \mu(y) d \mu(x) \\
& \leq \int_{B} \int_{S}\left|\mathscr{M}\left(f_{2}\right)(x)-\mathscr{M}\left(f_{2}\right)(y)\right| \mathrm{d} \mu(y) \mathrm{d} \mu(x) \\
& \quad+\mu(B) \int_{S} \mathscr{M}\left(f_{1}\right)(y) \mathrm{d} \mu(y) \\
& \quad+\mu(S) \int_{B} \mathscr{M}\left(f_{1}\right)(y) \mathrm{d} \mu(y) .
\end{aligned}
$$

Applying Hölder's inequality, [10, Corollary 6.3], and $L^{2}(\mu)$-boundedness of $\mathscr{M}$, we deduce that

$$
\begin{aligned}
& \mu(B) \int_{S} \mathscr{M}\left(f_{1}\right)(y) \mathrm{d} \mu(y) \\
& \leq \mu(B)[\mu(S)]^{1 / 2}\left(\int_{\mathscr{X}}\left[\mathscr{M}\left(f_{1}\right)(x)\right]^{2} \mathrm{~d} \mu(y)\right)^{1 / 2} \\
& \leq C \mu(B)[\mu(S)]^{1 / 2}\left(\int_{X}\left|f_{1}(y)\right|^{2} \mathrm{~d} \mu(y)\right)^{1 / 2} \\
& \quad=C \mu(B)[\mu(S)]^{1 / 2}\left(\int_{6 S}\left|f(y)-f_{6 S}\right|^{2} \mathrm{~d} \mu(y)\right)^{1 / 2} \\
& \leq C \mu(B)[\mu(S)]^{1 / 2}[\mu(36 S)]^{1 / 2}\|f\|_{\mathrm{RBMO}(\mu)} \\
& \leq C \mu(B) \mu(36 S)\|f\|_{\mathrm{RBMO}(\mu)} .
\end{aligned}
$$

There exists a nonnegative integer $m$ such that

$$
6^{m} B \subset 6 S \subset 6^{m+2}, \quad 36 S \subset 6^{m+3} B .
$$

Set $f_{4}:=\left(f-f_{6^{m} B}\right) \chi_{6^{m} B}$; then we get

$$
f_{1}-f_{4}=\left(f-f_{6 S}\right) \chi_{6 S \backslash 6^{m} B}+\left(f_{6^{m} B}-f_{6 S}\right) \chi_{6^{m} B} .
$$

For any $x \in B$, by applying the Minkowski inequality and a similar argument to (29) and (9), we have that

$$
\begin{aligned}
& \mathscr{M}\left(\left(f-f_{6 S}\right) \chi_{6 S \backslash 6^{m} B}\right)(x) \\
& \leq \int_{6 S \backslash 6^{m} B}|K(x, y)|\left|f(y)-f_{6 S}\right|\left(\int_{d(x, y)}^{\infty} \frac{\mathrm{d} t}{t^{3}}\right)^{1 / 2} \mathrm{~d} \mu(y) \\
& \leq C \int_{6 S \backslash 6^{m} B} \frac{1}{\lambda(x, d(x, y))}\left|f(y)-f_{6 S}\right| \mathrm{d} \mu(y) \\
& \leq C \frac{\mu\left(6^{m+3} B\right)}{\lambda\left(c_{B}, 6^{m} r_{B}\right)}\|f\|_{\mathrm{RBMO}(\mu)} \\
& \leq C\|f\|_{\mathrm{RBMO}(\mu)} .
\end{aligned}
$$

By using Definition 8 and Lemma 12, we can get

$\left|f_{6^{m} B}-f_{6 S}\right| \leq C K_{6^{m} B, 6^{m+3} B}\|f\|_{\operatorname{RBMO}(\mu)} \leq C\|f\|_{\operatorname{RBMO}(\mu)}$. 
Denote $a:=f_{6^{m} B}-f_{65}$. Let us estimate $\mathscr{M}\left(a \chi_{6^{m} B}\right)(x)$ :

$$
\begin{aligned}
& \mathscr{M}\left(a \chi_{6^{m} B}\right)(x) \\
& =\left(\int_{0}^{\infty}\left|\int_{d(x, y) \leq t} K(x, y) a \chi_{6^{m} B}(y) \mathrm{d} \mu(y)\right|^{2} \frac{\mathrm{d} t}{t^{3}}\right)^{1 / 2} .
\end{aligned}
$$

There are three cases about the relation of $6^{m} B$ and $\{y \in$ $x: d(x, y) \leq t\}$.

Case I. Consider $\{y \in \mathscr{X}: d(x, y) \leq t\} \subset 6^{m} B$; then by using the vanishing condition (14), we have

$$
\int_{d(x, y) \leq t} K(x, y) a \chi_{6^{m} B}(y) \mathrm{d} \mu(y)=0 .
$$

Case II. Consider $6^{m} B \subset\{y \in \mathscr{X}: d(x, y) \leq t\}$; then by using the vanishing condition (14), we have

$$
\begin{aligned}
& -\int_{d(x, y) \leq t} K(x, y) a \chi_{6^{m} B}(y) \mathrm{d} \mu(y) \\
& \quad=-\int_{6^{m} B} K(x, y) a \mathrm{~d} \mu(y) \\
& \quad=\int_{\left\{y \in X: d\left(x_{B}, y\right) \leq 6^{m} \cdot 3 r_{B}\right\} \backslash 6^{m} B} K(x, y) a \mathrm{~d} \mu(y) .
\end{aligned}
$$

Case III. Others. By applying the vanishing condition (14), we also get

$$
\begin{aligned}
& \int_{d(x, y) \leq t} K(x, y) a \chi_{6^{m} B}(y) \mathrm{d} \mu(y) \\
& \quad=-\int_{\{y \in X: d(x, y) \leq t\} \backslash 6^{m} B} K(x, y) a \mathrm{~d} \mu(y) .
\end{aligned}
$$

And, in this case, it is easy to get that $\{y \in \mathscr{X}: d(x, y) \leq$ $t\} \subset\left\{y \in \mathscr{X}: d\left(x_{B}, y\right) \leq 6^{m} 6 r_{B}\right\}$.

From this and the Minkowski inequality, it follows that

$$
\begin{aligned}
& \mathscr{M}\left(a \chi_{6^{m} B}\right)(x) \\
& =\left(\int_{0}^{\infty}\left|\int_{d(x, y) \leq t} K(x, y) a \chi_{6^{m} B}(y) \mathrm{d} \mu(y)\right|^{2} \frac{\mathrm{d} t}{t^{3}}\right)^{1 / 2} \\
& \leq C\|f\|_{\operatorname{RBMO}(\mu)} \int_{6^{m+1} B \backslash 6^{m} B} \frac{1}{\lambda(x, d(x, y))} \mathrm{d} \mu(y) \\
& \leq C\|f\|_{\operatorname{RBMO}(\mu)} .
\end{aligned}
$$

From (31), (32), and (38), we deduce that

$$
\int_{B} \mathscr{M}\left(f_{1}-f_{4}\right)(y) \mathrm{d} \mu(y) \leq C \mu(B)\|f\|_{\mathrm{RBMO}(\mu)} .
$$

On the other hand,

$$
\begin{aligned}
& f_{4}=\left(f-f_{6^{m} B}\right) \chi_{6^{m} B} \\
&=\left(f-f_{6 B}\right) \chi_{6 B}+\sum_{j=1}^{m-1}\left[\left(f-f_{6^{j+1} B}\right) \chi_{6^{j+1} B \backslash 6^{j} B}\right. \\
&\left.\quad-\left(f_{6^{j} B}-f_{6^{j+1} B}\right) \chi_{6^{j} B}\right] .
\end{aligned}
$$

By applying a similar argument of (29), (32), and (38), we have

$$
\int_{B} M\left(\left(f-f_{6 B}\right) \chi_{6 B}\right)(y) \mathrm{d} \mu(y) \leq C \mu(36 B)\|f\|_{\mathrm{RBMO}(\mu)},
$$

$\mathscr{M}\left(\left(f-f_{6^{j+2} B}\right) \chi_{6^{j+2} B} \backslash 6^{j} B\right) \leq C \frac{\mu\left(6^{j+2} B\right)}{\lambda\left(c_{B}, 6^{j+2} r_{B}\right)}\|f\|_{\operatorname{RBMO}(\mu)}$,

$$
\mathscr{M}\left(\left(f_{6^{j} B}-f_{6^{j+2} B}\right) \chi_{6^{j} B}\right) \leq C \frac{\mu\left(6^{j+2} B\right)}{\lambda\left(c_{B}, 6^{j+2} r_{B}\right)}\|f\|_{\operatorname{RBMO}(\mu)} .
$$

From the above inequalities, it follows that

$$
\int_{B} \mathscr{M}\left(f_{4}\right)(x) \mathrm{d} \mu(x) \leq C \mu(36 B) K_{B, S}\|f\|_{\mathrm{RBMO}(\mu)},
$$

and by (39), we deduce that

$$
\mu(S) \int_{S} \mathscr{M}\left(f_{1}\right)(x) \mathrm{d} \mu(x) \leq C \mu(S) \mu(36 B) K_{B, S}\|f\|_{\mathrm{RBMO}(\mu)} .
$$

Now let us estimate

$$
\left|\mathscr{M}\left(f_{2}\right)(x)-\mathscr{M}\left(f_{2}\right)(y)\right| \leq C\|f\|_{\mathrm{RBMO}(\mu)} .
$$

To prove (44), suppose

$$
D_{1}:=\left(\int_{0}^{\infty}\left[\int_{d(y, z) \leq t \leq d(x, z)}|K(x, z)|\left|f_{2}(z)\right| \mathrm{d} \mu(z)\right]^{2} \frac{\mathrm{d} t}{t^{3}}\right)^{1 / 2}
$$

$D_{2}:=\left(\int_{0}^{\infty}\left[\int_{d(x, z) \leq t \leq d(y, z)}|K(y, z)|\left|f_{2}(z)\right| \mathrm{d} \mu(z)\right]^{2} \frac{\mathrm{d} t}{t^{3}}\right)^{1 / 2}$,

$$
\begin{gathered}
D_{3}:=\left(\int _ { 0 } ^ { \infty } \left[\int_{\max (d(x, z), d(y, z))<t}|K(x, z)-K(y, z)|\right.\right. \\
\left.\left.\cdot\left|f_{2}(z)\right| \mathrm{d} \mu(z)\right]^{2} \frac{\mathrm{d} t}{t^{3}}\right)^{1 / 2} .
\end{gathered}
$$


Now we estimate $\left|\mathscr{M}\left(f_{2}\right)(x)-\mathscr{M}\left(f_{2}\right)(y)\right|$,

$$
\begin{aligned}
& \left|\mathscr{M}\left(f_{2}\right)(x)-\mathscr{M}\left(f_{2}\right)(y)\right| \\
& =\mid\left(\left.\int_{0}^{\infty}\left|\int_{d(x, z) \leq t} K(x, z) f_{2}(z)\right| \mathrm{d} \mu(z)\right|^{2} \frac{\mathrm{d} t}{t^{3}}\right)^{1 / 2} \\
& \quad-\left(\int_{0}^{\infty}\left|\int_{d(y, z) \leq t} K(y, z) f_{2}(z) \mathrm{d} \mu(z)\right|^{2} \frac{\mathrm{d} t}{t^{3}}\right)^{1 / 2} \mid \\
& \leq\left(\int_{0}^{\infty} \mid \int_{d(x, z) \leq t} K(x, z) f_{2}(z) d \mu(z)\right. \\
& \quad \leq \sum_{i=1}^{3} D_{i} .
\end{aligned}
$$

For $x, y \in S$, applying the Minkowski inequality and (9), we can deduce that

$$
\begin{aligned}
& D_{1} \leq \int_{d(y, z) \leq d(x, z)} \frac{d(x, z)}{\lambda(x, d(x, z))}\left(\int_{d(y, z)}^{d(x, z)} \frac{\mathrm{d} t}{t^{3}}\right)^{1 / 2} \mathrm{~d} \mu(z) \\
& \leq C \int_{d(y, z) \leq d(x, z)} \frac{\left|f_{2}(z)\right|}{\lambda(x, d(x, z))}\left[\frac{d(x, y)}{d(x, z)}\right]^{1 / 2} \mathrm{~d} \mu(z) \\
& \leq C r_{S}^{1 / 2} \int_{\mathscr{X} \backslash 6 S} \frac{\left|f(z)-f_{6 S}\right|}{\lambda(x, d(x, z))} \frac{\mathrm{d} \mu(z)}{[d(x, z)]^{1 / 2}} \\
& \leq C r_{S}^{1 / 2} \sum_{k=1}^{\infty} \int_{6^{k+1} S \backslash 6^{k} S} \frac{\left|f(z)-f_{6 S}\right|}{\lambda(x, d(x, z))} \frac{\mathrm{d} \mu(z)}{[d(x, z)]^{1 / 2}} \\
& \leq C r_{S}^{1 / 2} \sum_{k=1}^{\infty} \frac{\mu\left(6^{k+1} S\right)}{\lambda\left(c_{S}, 6^{k+1} S\right)} \frac{1}{\left(6^{k} r_{S}\right)^{1 / 2}} \frac{1}{\mu\left(6^{k+1} S\right)} \\
& \cdot \int_{6^{k+1} S}\left|f(z)-f_{6 S}\right| \mathrm{d} \mu(z) \\
& \leq C \sum_{k=1}^{\infty} 6^{-k / 2} \frac{1}{\mu\left(6^{k+1} S\right)} \\
& \cdot \int_{6^{k+1} S}\left[\left|f(z)-f_{6^{k+1} S}\right|+\left|f_{6^{k+1} S}-f_{6 S}\right|\right] \mathrm{d} \mu(z) \\
& \leq C \sum_{k=1}^{\infty} 6^{-k / 2}(k+1)\|f\|_{\operatorname{RBMO}(\mu)} \\
& \leq C\|f\|_{\operatorname{RBMO}(\mu)} \text {. }
\end{aligned}
$$

For $D_{2}$, by using a similar method of $D_{1}$, we have

$$
D_{2} \leq C\|f\|_{\operatorname{RBMO}(\mu)} .
$$

Now let us estimate $D_{3}$; by applying (13), we obtain

$$
\begin{aligned}
& D_{3} \leq \int_{\mathscr{X}}|K(x, z)-K(y, z)|\left|f_{2}(z)\right| \\
& \cdot\left(\int_{\max (d(x, z), d(y, z)) \leq t} \frac{\mathrm{d} t}{t^{3}}\right)^{1 / 2} \mathrm{~d} \mu(z) \\
& \leq C \int_{\mathscr{X} \backslash 6 S} \frac{[d(x, y)]^{\delta}[d(x, z)]^{1-\delta}}{\lambda(x, d(x, z))} \\
& \cdot\left|f(z)-f_{6 S}\right| \frac{1}{d(x, z)} \mathrm{d} \mu(z) \\
& \leq C \int_{\mathscr{X} \backslash 6 S} \frac{[d(x, y)]^{\delta}[d(x, z)]^{-\delta}}{\lambda(x, d(x, z))}\left|f(z)-f_{6 S}\right| \mathrm{d} \mu(z) \\
& \leq C r_{S}^{\delta} \sum_{k=1}^{\infty} \int_{6^{k+1} S \backslash 6^{k} S} \frac{\left|f(z)-f_{6 S}\right|}{\lambda(x, d(x, z))} \frac{1}{[d(x, z)]^{\delta}} \mathrm{d} \mu(z) \\
& \leq C r_{S}^{\delta} \sum_{k=1}^{\infty} \frac{\mu\left(6^{k+1} S\right)}{\lambda\left(x_{S}, r_{6^{k} S}\right)} \frac{1}{\left(6^{k} r_{S}\right)^{\delta}} \frac{1}{\mu\left(6^{k+1} S\right)} \\
& \cdot \int_{6^{k+1} S}\left|f(z)-f_{6 S}\right| \mathrm{d} \mu(z) \\
& \leq C \sum_{k=1}^{\infty} 6^{-k \delta} \frac{1}{\mu\left(6^{k+1} S\right)} \\
& \cdot \int_{6^{k+1} S}\left[\left|f(z)-f_{6^{k+1} S}\right|+\left|f_{6^{k+1} S}-f_{6 S}\right|\right] \mathrm{d} \mu(z) \\
& \leq C\|f\|_{\operatorname{RBMO}(\mu)} \text {. }
\end{aligned}
$$

Combining the $D_{1}, D_{2}$, and $D_{3}$, we know that (44) holds. And then we have

$$
\begin{aligned}
& \int_{B} \int_{S}\left|\mathscr{M}\left(f_{2}\right)(x)-\mathscr{M}\left(f_{2}\right)(y)\right| \mathrm{d} \mu(y) \mathrm{d} \mu(x) \\
& \quad \leq C \mu(B) \mu(S)\|f\|_{\mathrm{RBMO}(\mu)} .
\end{aligned}
$$

Finally, we obtain that, for any two balls $B \subset S$,

$$
\begin{aligned}
& \int_{B} \int_{S}|\mathscr{M}(f)(x)-\mathscr{M}(f)(y)| d \mu(y) \mathrm{d} \mu(x) \\
& \quad \leq C K_{B, S}\|f\|_{\mathrm{RBMO}(\mu)}(\mu(B) \mu(36 S)+\mu(36 B) \mu(S)) .
\end{aligned}
$$

Suppose that $B=S$ is a $\left(36, \beta_{36}\right)$-doubling ball; by (51) we have

$$
\begin{aligned}
& \frac{1}{\mu(B)} \int_{B}\left|\mathscr{M}(f)(x)-m_{B}(\mathscr{M}(f))\right| \mathrm{d} \mu(x) \\
& \quad \leq \frac{1}{\mu(B) \mu(B)} \int_{B} \int_{B}|\mathscr{M}(f)(x)-\mathscr{M}(f)(y)| \mathrm{d} \mu(y) \mathrm{d} \mu(x) \\
& \quad \leq C\|f\|_{\operatorname{RBMO}(\mu)} .
\end{aligned}
$$


And when $B \subset S$ are two $\left(36, \beta_{36}\right)$-doubling balls, it can be deduced that

$$
\begin{aligned}
& \left|m_{B}(\mathscr{M}(f))-m_{S}(\mathscr{M}(f))\right| \\
& \quad \leq \frac{1}{\mu(B) \mu(S)} \int_{B} \int_{S}|\mathscr{M}(f)(x)-\mathscr{M}(f)(y)| \mathrm{d} \mu(y) \mathrm{d} \mu(x) \\
& \quad \leq C\|f\|_{\operatorname{RBMO}(\mu)} K_{B, S} .
\end{aligned}
$$
have

Applying Lemma 13 and the above two inequalities, we

$$
\|\mathscr{M}(f)\|_{\mathrm{RBMO}(\mu)} \leq C\|f\|_{\mathrm{RBMO}(\mu)} .
$$

If $f \in \operatorname{RBMO}(\mu)$ such that $\mathscr{M}(f)$ is infinite on a set of positive measures, it is easy to prove that we take a ball $B$ such that

$$
\int_{B} \mathscr{M}(f)(x)=\infty
$$

namely, $\mathscr{M}(f)(x) \notin L_{\text {loc }}(\mu)$. So, $\mathscr{M}(f)(x) \notin \operatorname{RBMO}(\mu)$.

We complete the proof of Theorem 11.

\section{Conflict of Interests}

The authors declare that they do not have any commercial or associative interest that represents a conflict of interests in connection with the work submitted.

\section{Acknowledgments}

This work is supported by National Science Foundation of China (Grant no. 11261055) and College Students' Innovative Project of Xinjiang University (Grant no. 201410755008).

\section{References}

[1] J. Marcinkiewicz, "Sur quelques intégrales du type de Dini," Annales de la Société Polonaise de Mathématique, vol. 17, pp. 4250, 1938.

[2] A. Zygmund, "On certain integrals," Transactions of the American Mathematical Society, vol. 55, pp. 170-204, 1944.

[3] E. M. Stein, "On the functions of Littlewood-Paley, LUSin, and Marcinkiewicz," Transactions of the American Mathematical Society, vol. 88, pp. 430-466, 1958.

[4] Y. Ding, Q. Xue, and K. Yabuta, "A remark to the $L^{2}$ boundedness of parametric Marcinkiewicz integral," Journal of Mathematical Analysis and Applications, vol. 387, pp. 691-697, 2012.

[5] Y. Ding, S. Lu, and P. Zhang, "Weighted weak type estimates for commutators of the Marcinkiewicz integrals," Journal of Mathematical Analysis and Applications, vol. 47, pp. 83-95, 2012.

[6] R. R. Coifman and G. Weiss, Analyse Harmonique NonCommutative sur Certains Espaces Homogènes, vol. 242 of Lecture Notes in Mathematics, Springer, Berlin, Germany, 1971.

[7] X. Tolsa, "Painlevé's problem and the semiadditivity of analytic capacity," Acta Mathematica, vol. 190, no. 1, pp. 105-149, 2003.

[8] G. Hu, H. Lin, and D. Yang, "Marcinkiewicz integrals with nondoubling measures," Integral Equations and Operator Theory, vol. 58, no. 2, pp. 205-238, 2007.
[9] S. Wang, Y. Jiang, and B. Li, "Boundedness of Marcinkiewicz integrals on RBMO," Advances in Mathematics, vol. 41, no. 4, pp. 447-454, 2012.

[10] T. Hytönen, "A framework for non-homogeneous analysis on metric spaces, and the RBMO space of Tolsa," Publicacions Matemàtiques, vol. 54, no. 2, pp. 485-504, 2010.

[11] D. Yang, D. Yang, and G. Hu, The Hardy Space $\mathrm{H}^{1}$ with NonDoubling Measures and Their Applications, vol. 2084 of Lecture Notes in Mathematics, Springer, Berlin , Germany, 2013.

[12] T. A. Bui and X. T. Duong, "Hardy spaces, regularized BMO and the boundedness of Caldeŕn-Zymgund operators on homogeneous spaces," Journal of Geometric Analysis, vol. 23, no. 2, pp. 895-932, 2013.

[13] X. Tolsa, " $B M O, H^{1}$, and Calderón-Zygmund operators for non doubling measures," Mathematische Annalen, vol. 319, no. 1, pp. 89-149, 2001.

[14] H. Lin and D. Yang, "Equivalent boundedness of Marcinkiewicz integrals on non-homogeneous metric measure spaces," Science China, vol. 57, no. 1, pp. 123-144, 2014.

[15] X. Fu and D. Yang, "The molecular characterization of the Hardy space $H^{1}$ on non-homogeneous metric measure spaces and its application," Journal of Mathematical Analysis and Applications, vol. 410, no. 2, pp. 1028-1042, 2014.

[16] T. Hytönen, D. Yang, and D. Yang, "The Hardy space $H^{1}$ on nonhomogeneous metric spaces," Mathematical Proceedings of the Cambridge Philosophical Society, vol. 153, no. 1, pp. 9-31, 2012.

[17] D. Yang, D. Yang, and X. Fu, "The Hardy space $H^{1}$ on nonhomogeneous metric spaces and its applications-a survey," Eurasian Mathematical Journal, vol. 4, no. 2, pp. 104-139, 2013.

[18] H. Lin and D. Yang, "Spaces of type BLO on non-homogeneous metric measure," Frontiers of Mathematics in China, vol. 6, no. 2, pp. 271-292, 2011. 


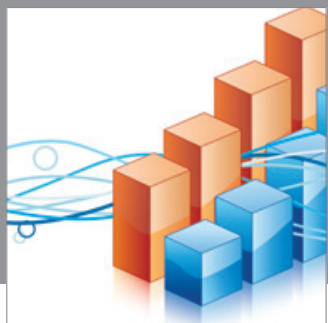

Advances in

Operations Research

mansans

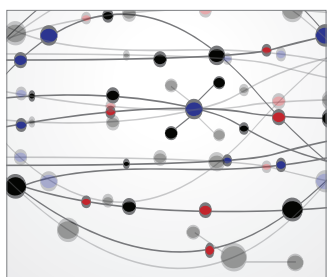

The Scientific World Journal
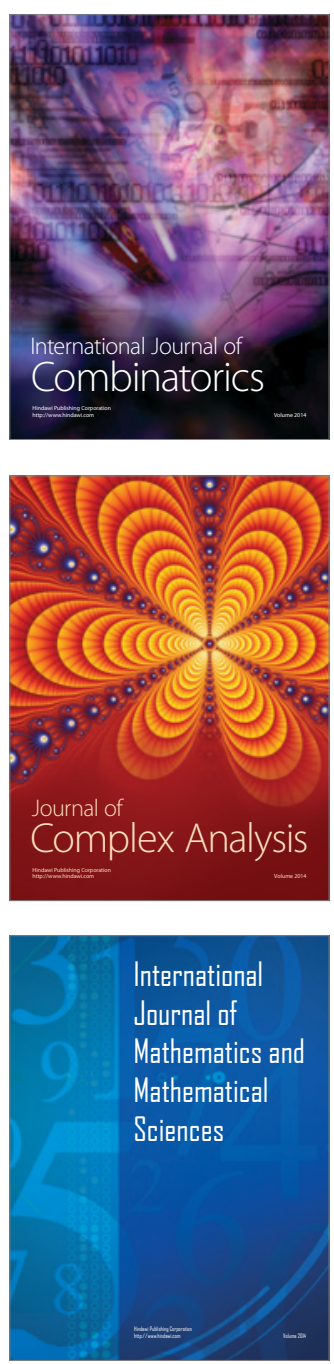
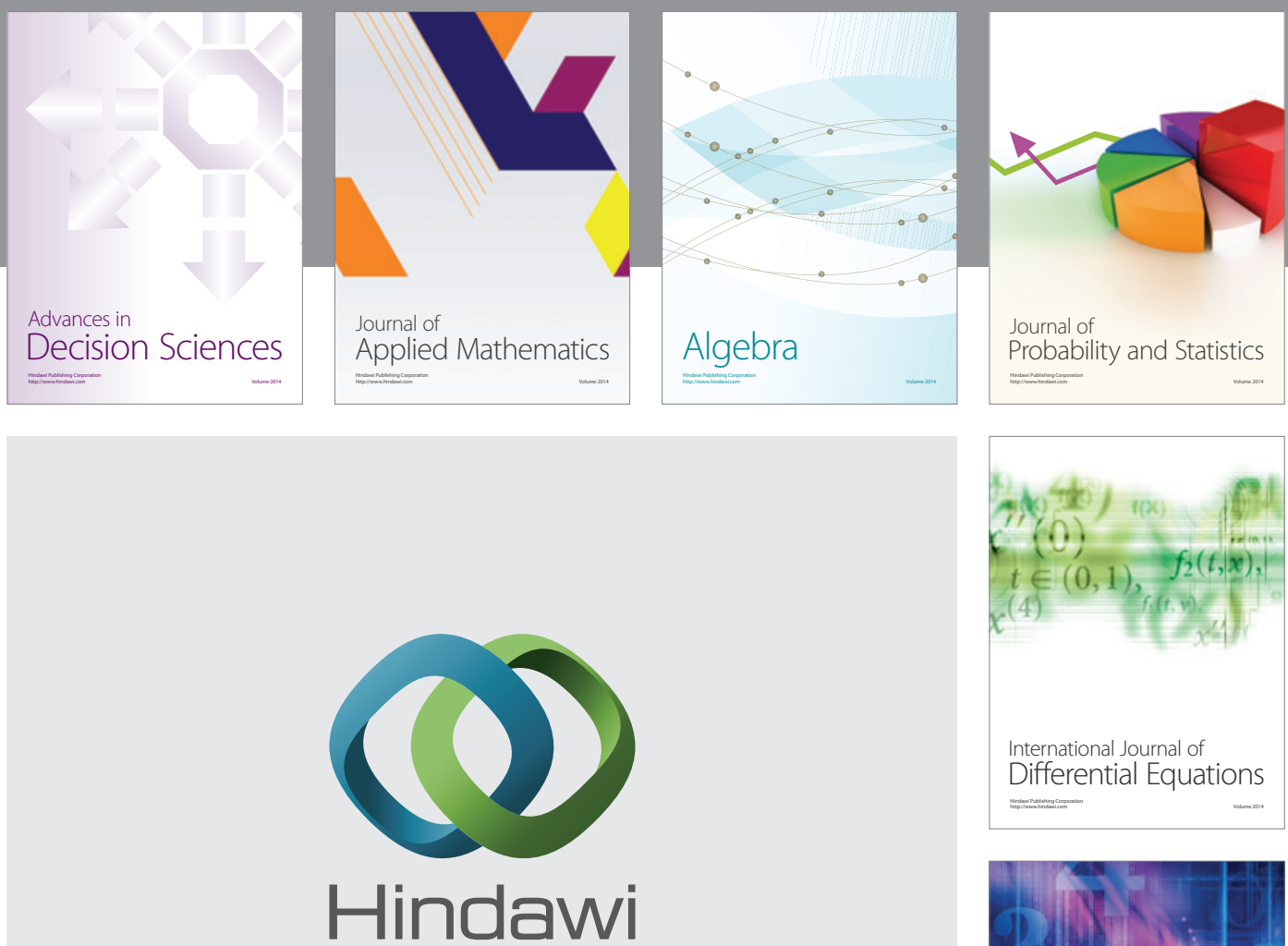

Submit your manuscripts at http://www.hindawi.com
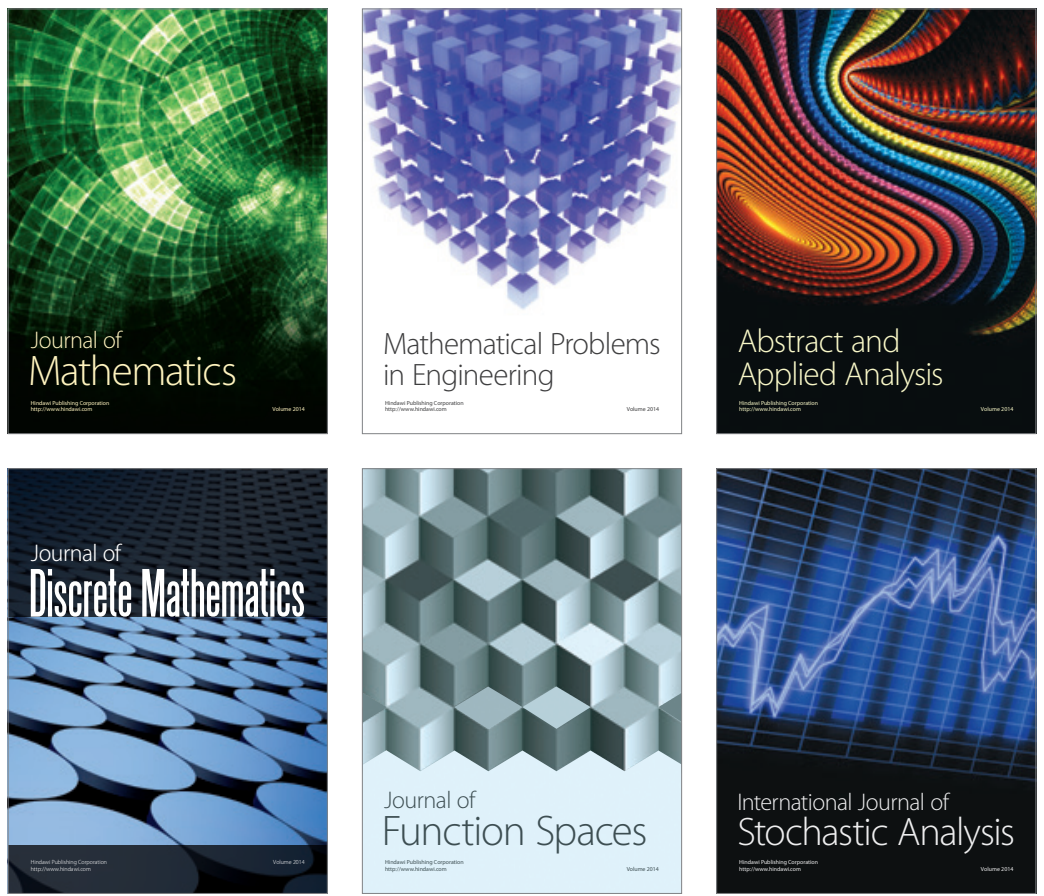

Journal of

Function Spaces

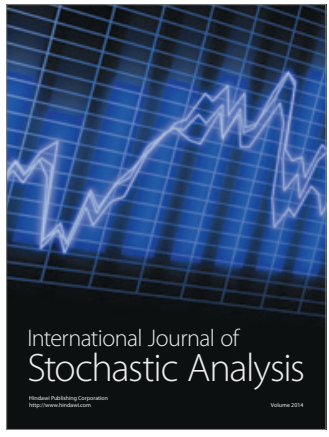

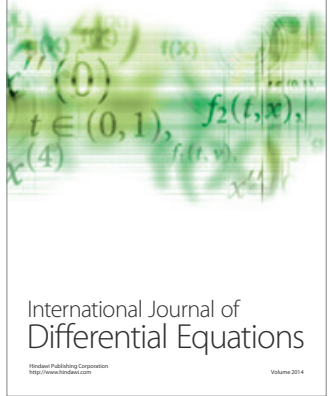
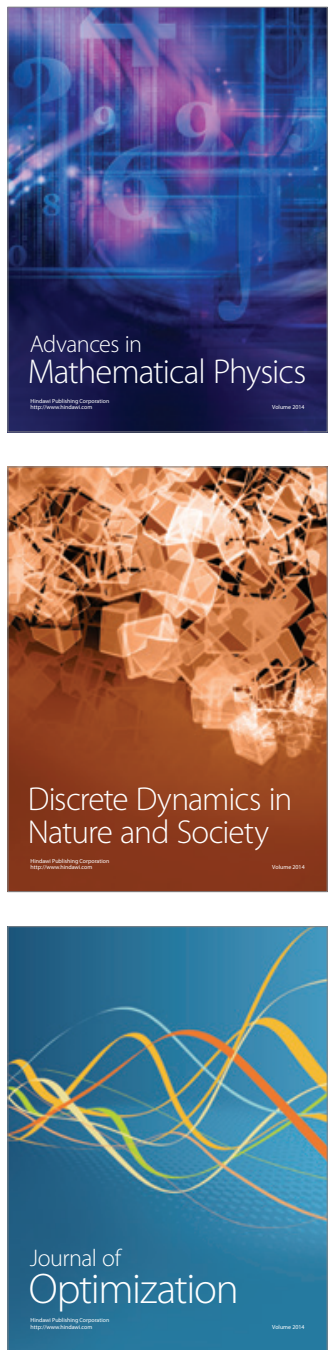